\title{
Interacción de las exigencias de trabajo en la generación de sufrimiento mental
}

\author{
Interaction of work demands \\ in the genesis of mental suffering
}

\begin{abstract}
Mariano Noriega 1 Cristina Laurell 1

Susana Martínez 1

Ignacio Méndez 2

Jorge Villegas 1
\end{abstract}

\footnotetext{
1 División de Ciencias Biológicas y de la Salud, Universidad Autónoma Metropolitana-Xochimilco. Calzada del Hueso 1100, Col. Villa Quietud, Delegación Coyoacán, México, D.F. 04960, México. mnoriega@cueyatl.uam.mx claurell@cueyatl.uam.mx smartin@cueyatl.uam.mx jvillegas@cueyatl.uam.mx 2 Instituto de Investigaciones en Matemáticas Aplicadas y en Sistemas, Universidad Nacional Autónoma de México. C. P. 01000, Administración no 20, Delegación Alvaro Obregón, México, D.F. 20726, México. imendez@servidor.unam.mx
}

\begin{abstract}
Current working conditions and new forms of work organization are affecting workers' health in numerous ways which can only be explained by more complex theories and methodologies than those used traditionally. The authors analyze some important elements of the work process and the interaction among work demands as determinants of mental and psychosomatic disorders and fatigue (MPDF) among workers in a Mexican industrial plant. The workers studied $(n=830)$ were male, with a mean age of 32. MPDF represent one-third of the disease burden among these workers. An association was observed with the number of years worked, type of activity, and job area. Job-related demands and work organization, including excessive work, strict supervision, dangerous work, unnatural positions, and intense and hard physical labor were also closely related to these conditions. The most relevant problem is the combination of many different demands. Interaction among the combinations was found to be additive or synergistic. In the latter condition the risk of morbid effects increases beyond merely an additive effect.
\end{abstract}

Key words Mental Disorders; Fatigue; Work

Resumen Las actuales condiciones de trabajo y las nuevas formas de organización laboral tienen diversos efectos en la salud de los trabajadores, lo que obliga a explicarlos con teorías y metodologías más complejas que las tradicionales. Se analizan algunos elementos del proceso de trabajo y la interacción de exigencias laborales como determinantes de trastornos mentales, psicosomáticos y fatiga (TMPF) en trabajadores de una industria mexicana. Los obreros estudiados $(n=830)$ fueron del sexo masculino, con promedio de edad de 32 años. Los TMPF representan una tercera parte del total de la patología de estos trabajadores. Se encontró asociación con el tipo de actividad y el área de trabajo. Las exigencias derivadas del trabajo y de la organización laboral tales como demasiado trabajo, supervisión estricta, trabajo peligroso, posiciones forzadas y esfuerzo físico intenso muestran también una estrecha relación con estos padecimientos y, en menor medida, la rotación de turnos y el doble turno. El problema encontrado como más relevante fue la combinación de múltiples exigencias. Estas combinaciones se manifestaron en forma de interacción sinérgica o aditiva; la primera potencia el riesgo de que suceda un efecto mórbido, mientras que la segunda lo suma.

Palabras clave Trastornos Mentales; Fatiga; Trabajo 


\section{Introducción}

Desde hace varias décadas en los países industrializados se identificó la importancia que tiene el trabajo en la generación de trastornos mentales y psicosomáticos (Gardell, 1971). En el transcurso de varios años y desde diversas perspectivas, se hicieron notar las consecuencias de la organización del trabajo y el estrés sobre la salud y el bienestar de los trabajadores. También el impacto de este fenómeno sobre la economía mostró ser de una enorme magnitud (Karasek \& Theorell, 1990; Creed, 1993).

En países de América Latina, el interés por tal problemática también ha estado presente desde hace varios años (Henao, 1982; Seligmann, 1986) y resulta de interés en la actualidad, si se considera el efecto que la modernización y la globalización económica han producido sobre las condiciones laborales de los trabajadores, ya que para muchos se han incrementado los riesgos y exigencias de trabajo como resultado de los cambios tecnológicos y sobre todo por las formas de organización del trabajo a que hoy se enfrentan (Sánchez \& González, 1997).

México no ha sido ajeno a esta situación, y se encuentran numerosos ejemplos de modernización que han pasado por la reconversión tecnológica y la transformación de las relaciones laborales (Rueda, 1998). Esta estrategia, orientada ante todo a incrementar la productividad, inició hacia fines de la década de los ochenta y se caracterizó por la flexibilización en el uso de la fuerza de trabajo con la consecuente disminución del control obrero sobre el proceso laboral y la intensificación del trabajo. Esto determinó que se conformaran perfiles de exigencias laborales que se han asociado con un incremento en los problemas de salud física y mental de los trabajadores (Laurell et al., 1991).

El análisis de los problemas de salud derivados de esta nueva situación representa un reto, porque su estudio exige ampliar el horizonte desde donde tradicionalmente se han abordado en nuestro país. En primer término porque significa explorar aspectos del trabajo, como las exigencias laborales, que no constituyen el punto de atención de la medicina del trabajo o de la psicología, disciplinas que hegemonizan la práctica de la salud en el trabajo en México, además de que la nueva situación existente obliga a pensar en fenómenos como la multiexposición laboral y los efectos que ello pudiera acarrear sobre la salud de los trabajadores.

En este artículo se analiza la información obtenida en un estudio realizado en una em- presa siderúrgica en México que puede considerarse ilustrativa de la situación que se enfrenta como producto de estos cambios en la organización del trabajo. Los estudios realizados en la industria siderúrgica en otros países han permitido identificar la complejidad del propio proceso y de las consecuencias que éste genera en los trabajadores. Particularmente se ha profundizado en los efectos que distintos aspectos de la organización laboral producen sobre la salud física y mental de los trabajadores. Para ello han analizado tanto aspectos cualitativos como cuantitativos del trabajo.

De manera sintética, se puede señalar que estudios realizados acerca de las jornadas extensas o con sobrecarga cuantitativa de trabajo parecen mostrar un efecto moderado sobre la salud mental de los trabajadores. También las jornadas extensas se han relacionado con un mayor riesgo de infarto del miocardio, hipertensión y diabetes. Por otra parte, aquellos estudios que analizan el control sobre el trabajo o aspectos relacionados con la descalificación de actividades y demandas de carácter cualitativo muestran una mayor asociación con efectos por estrés, así como situaciones adictivas como el alcoholismo. Igualmente las demandas de orden cualitativo han mostrado una fuerte asociación con problemas psiquiátricos como la depresión (Kawakami \& Haratami, 1999).

También trabajadores de la industria pesada han mostrado efectos en la salud al estudiarse problemas de la organización del trabajo. Los obreros de menor antigüedad son más susceptibles de sufrir algún trastorno mental y aquéllos con altas demandas estarían en peores condiciones de salud. Por el contrario, entre mayor capacidad de decisión en el trabajo y mejor entorno, los trabajadores se encontrarían en mejores condiciones. Así se ha establecido un perfil que muestra que aquéllos que han trabajado más tiempo y tienen mayor capacidad de decisión y mejor entorno laboral (social support) tienen un menor riesgo de padecer trastornos mentales (Yang et al., 1997).

En trabajadores de otros procesos industriales, como en la electrónica, se han establecido relaciones entre el estrés laboral y trastornos mentales. Las causas del estrés radican en aspectos de orden cualitativo como: presencia de conflictos en el trabajo, responsabilidad excesiva, exigencia de no cometer errores, mala relación con los supervisores, así como dificultad de mantenerse al tanto de los cambios en la tecnología (Shigemi et al., 1997).

Llama la atención que también los aspectos subjetivos del puesto, en estudios realizados con trabajadores de servicios, tengan una 
mayor asociación con los trastornos mentales que las condiciones objetivas del mismo (Goldberg et al., 1996). Cabe mencionar que, en este último estudio, a diferencia de lo encontrado por Yang (Yang et al., 1997), en este caso la antigüedad se asoció con síntomas depresivos y ansiedad entre varones.

Los planteamientos centrales que se encuentran en la base de este artículo señalan al proceso de trabajo como una categoría explicativa y determinante de la salud de los trabajadores en el cual los riesgos y exigencias laborales actúan como mediadores entre el trabajo y los trastornos mentales, psicosomáticos y fatiga (TMPF). Interesa realizar el análisis considerando la exposición múltiple a tales exigencias y explorar la presencia de un mayor número de daños a la salud como resultado de las condiciones de tal multiexposición.

Para identificar los perfiles de exigencias y de TMPF, se comparan grupos con diferente inserción en el proceso laboral: trabajadores vinculados directamente a la producción y trabajadores que realizan actividades administrativas.

\section{Metodología}

El estudio se llevó a cabo en un grupo de trabajadores varones $(n=830)$ de una empresa siderúrgica. El $80 \%$ tenía menos de 40 años y su promedio de edad era de 32 años, con una desviación estándar de 10,4.

Se aplicó una encuesta para uso epidemiológico que incluía la recolección de información sobre exposición a diversas exigencias laborales. Las preguntas se hicieron en un lenguaje apropiado para los obreros, de acuerdo a una indagación previa de cómo se entendía cada problema y qué significaba para cada trabajador.

La sección de morbilidad de la encuesta se construyó para captar sintomatología de los daños a la salud relacionados con el proceso de trabajo. Incluía dos índices. El Índice Médico Cornell (Abramxon, 1964) y el Índice de Yoshitake (Yoshitake, 1978). El primero permite establecer diagnósticos presuntivos de algunos trastornos mentales y psicosomáticos. Para este estudio, se valoró el formulario en su conjunto y tres problemas específicos: depresión, ansiedad y trastornos psicosomáticos. El segundo capta la presencia o ausencia de fatiga por medio de una encuesta validada para el trabajo industrial (Couto et al., 1981; Almirall \& Reyes, 1982).

La codificación de la información acerca de los trastornos mentales se realizó de acuerdo a lo establecido en el DSM-III Manual diagnós- tico y estadístico de los trastornos mentales (APA/OPS, 1988).

La forma en que se agrupó la patología fue la siguiente: a) Trastornos mentales: irritabilidad, ansiedad y depresión; b) Trastornos del sueño; c) Trastornos psicosomáticos digestivos: gastritis, úlcera gástrica y úlcera duodenal; d) Trastornos psicosomáticos cardiovasculares: hipertensión y enfermedad isquémica del corazón; e) Trastornos neurológicos: cefalea tensional y migraña; y f) Fatiga.

Sólo se consideró un diagnóstico como máximo para cada persona, en cada uno de esos conjuntos patológicos.

Para el procesamiento de la información, se controlaron la edad y la antigüedad de los trabajadores y se utilizó el análisis multivariado, en particular los modelos logísticos. Se analizaron los diagnósticos en relación con el tipo de actividad, las áreas, la antigüedad en el trabajo y las exigencias laborales. En un segundo momento, se analizó la interacción de dos exigencias con los trastornos mencionados, con la finalidad de explorar el fenómeno de la multiexposición y de los efectos que ésta puede estar ocasionando sobre el perfil de morbilidad de los trabajadores.

\section{Resultados}

El análisis de la información permite mostrar tres manifestaciones distintas del impacto del proceso laboral en los trastornos mentales, los psicosomáticos y la fatiga (TMPF) de los trabajadores. El primer aspecto confirma la conocida asociación de algunas variables del trabajo, tales como el tipo de actividad, el área de trabajo y la antigüedad en relación con los TMPF. El segundo muestra la relación de estos trastornos con algunas exigencias laborales, la mayoría de ellas poco estudiadas en la salud laboral. Por último, el tercer aspecto aborda un problema novedoso en este campo como es el estudio de la interacción y la aditividad de las exigencias en la generación de los TMPF.

\section{El trabajo}

El tipo de actividad que realizan los trabajadores es fundamental en la aparición de TMPF. El promedio de probabilidades de que aparezcan estos padecimientos, ajustado por edad y antigüedad, presenta una marcada diferencia entre los trabajadores de producción en relación con los administrativos (Tabla 1). La fatiga es el trastorno más importante en los dos grupos, pero con una frecuencia casi del doble en los 
primeros con respecto a los segundos. Estas diferencias se han encontrado en otros estudios que comparan obreros y empleados (Aro \& Hasan, 1987). Los trastornos psicosomáticos cardiovasculares son tres veces más frecuentes en los de producción y, en el resto de padecimientos estudiados, el promedio de probabilidades más alto se presenta también entre los trabajadores que se encuentran directamente en la producción, duplicándose en el caso de las cefaleas y los trastornos del sueño.

Esto se debe a que los trabajadores de producción están expuestos a una amplia gama de exigencias y, casi siempre, sin posibilidad de protegerse contra ellas. Por el contrario, el trabajo administrativo se encuentra fuera de las áreas de producción e implica menor exposición a las exigencias que impone la siderurgia. Las diferencias entre los dos grupos son signi-

Tabla 1

Trastornos mentales, psicosomáticos y fatiga según tipo de actividad.

\begin{tabular}{lccc}
\hline Diagnóstico & \multicolumn{2}{c}{ Probabilidad1 } & p \\
& producción & administración & * \\
\hline Fatiga & 0,503 & 0,315 & * \\
Psicosomáticos digestivos & 0,444 & 0,263 & * \\
Trastornos mentales & 0,357 & 0,263 & * \\
Cefalea y migraña & 0,388 & 0,184 & - \\
Trastornos de sueño & 0,354 & 0,184 & 0,052 \\
Psicosomáticos corazón & 0,170 & &
\end{tabular}

1 Promedio de probabilidad ajustado por edad y antigüedad.

$p={ }^{*} p<0,05$. Modelos logísticos.

Fuente: Encuesta individual.

Tabla 2

Trastornos mentales, psicosomáticos y fatiga por áreas específicas:

aceración y administración.

\begin{tabular}{|c|c|c|c|}
\hline \multirow[t]{2}{*}{ Diagnóstico } & \multicolumn{2}{|c|}{ Probabilidad 1} & \multirow[t]{2}{*}{$\mathrm{p}$} \\
\hline & aceración & administración & \\
\hline Fatiga & 0,558 & 0,316 & * \\
\hline Psicosomáticos digestivo & 0,483 & 0,263 & * \\
\hline Trastornos mentales & 0,458 & 0,263 & * \\
\hline Cefalea y migraña & 0,425 & 0,184 & ** \\
\hline Trastornos de sueño & 0,400 & 0,184 & * \\
\hline Psicosomáticos corazón & 0,116 & 0,053 & - \\
\hline
\end{tabular}

1 Promedio de probabilidades ajustado por edad y antigüedad.

$\mathrm{p}={ }^{\star} \mathrm{p}<0,05 ;{ }^{\star \star} \mathrm{p}<0,01$. Modelos logísticos.

Fuente: Encuesta Individual. ficativas para el conjunto de diagnósticos analizados, con excepción de los trastornos mentales y los psicosomáticos del corazón.

Las áreas o departamentos son las unidades más amplias en que se organiza la producción. Para ilustrar el efecto que implica el trabajar en un área determinada de este proceso, se comparó una de las áreas más expuestas de la siderúrgica, como es la de Aceración, con la de los trabajadores administrativos, como grupo "control".

Se evidencia en el Tabla 2 que el área de Aceración presenta una probabilidad de trastornos mentales y psicosomáticos de una magnitud mucho mayor en relación con los trabajadores administrativos. Los modelos logísticos señalan también diferencias significativas, con excepción de los trastornos psicosomáticos del corazón. En conclusión, el riesgo de trabajar, en una u otra área, dada la complejidad de sus procesos de trabajo y las exigencias a las que se someten estos trabajadores, se traduce, por lo menos, en alrededor del doble de riesgo de padecer TMPF.

Otro aspecto interesante surgido en este estudio es que el "tiempo de exposición" al trabajo, para la aparición de los TMPF, es bastante corto, al contrario de lo que sucede con la gran mayoría de las enfermedades reconocidas como profesionales. El análisis epidemiológico muestra que aproximadamente 4 de cada 10 trabajadores llegan a padecer, en su vida laboral, fatiga y algún trastorno mental y psicosomático. Las diferencias fueron estadísticamente significativas sólo para el caso de las cefaleas y las enfermedades cardiovasculares entre los trabajadores con más de 9 años de antigüedad y los de menos de 4 años. Este hallazgo apunta a que el tiempo de exposición en el puesto de trabajo no es una condición determinante para la aparición de los TMPF. Este diferencial comportamiento de otro tipo de enfermedades laborales en los trabajadores obliga a reflexionar sobre la importancia de una detección temprana y una prevención y tratamiento oportunos, paralelamente a su reconocimiento profesional. Otro hecho a reflexionarse es si estas semejanzas entre las poblaciones con corto y largo tiempo de exposición no se deben al conocido "efecto del trabajador sano", ampliamente discutido y difundido en la literatura científica (Wilcosky \& Wing, 1987).

\section{Las exigencias}

Las principales exigencias laborales estudiadas fueron: rotación de turnos, doble turno tres veces por semana o más, excesiva atención, rit- 
mo intenso, demasiado trabajo, trabajo aburrido (monotonía), supervisión estricta, situaciones inadecuadas de responsabilidad, emergencias, trabajo peligroso, esfuerzo físico intenso y posiciones forzadas e incómodas. Todas ellas están en estrecha relación con el tipo de actividad y con las características de la organización laboral (Hoiberg, 1982).

Se analizaron las diversas exigencias para cada una de las manifestaciones patológicas relacionadas con la salud mental. Los resultados (Tabla 3) evidencian una clara relación entre el conjunto de exigencias y los TMPF. La diferencia estadística entre los expuestos y los no expuestos es muy consistente en casi todo el conjunto de padecimientos estudiados.

Es muy notorio que, siendo la rotación de turnos una de las exigencias mayormente estudiadas por los científicos de la salud en el trabajo, dadas las consecuencias negativas que tiene a nivel psicofisiológico, prácticamente no haya presentado diferencias estadísticamente significativas con los padecimientos estudiados, con excepción de los trastornos del sueño. Este hallazgo llama la atención ya que ha sido consistentemente reportada en la literatura la relación que guarda esta exigencia con la fatiga, los psicosomáticos digestivos, del corazón y los trastornos mentales, principalmente (Akerstedt et al., 1984; Knutsson, 1986; Segawa et al., 1987).

Por otro lado, hay exigencias que tienen una relación más marcada con los problemas de salud mental. Esta situación se puede observar en el caso de aquéllas que implican mucha presión para los trabajadores, tales como el exceso de trabajo, la supervisión estricta y el trabajo peligroso. Estas exigencias presentan diferencias significativas con el conjunto de trastornos estudiados. Hallazgos similares se han encontrado con otro tipo de trabajadores que refieren una fuerte presión en el trabajo, en particular, con el tiempo de trabajo, estrés y enfermedades isquémicas del corazón (Stockmeier et al., 1989).

En el caso específico del trabajo aburrido, como una exigencia que tiene que ver con el contenido cualitativo del trabajo y que ha sido estudiada con menor frecuencia, presenta también una fuerte asociación con los cuadros patológicos estudiados.

\section{El sinergismo y la aditividad}

Probablemente el problema más relevante en procesos de trabajo complejos es la combinación de múltiples exigencias a las que se someten los trabajadores en las condiciones laborales modernas. Dependiendo de cómo se insertan los operarios en los procesos laborales, se pueden producir combinaciones que impactan de manera diferencial a éstos.

Para el análisis de esta información, se discuten el concepto de interacción sinérgica y el de no interacción que puede expresarse en el concepto de aditividad.

Trastornos mentales, psicosomáticos y fatiga en expuestos y no expuestos a exigencias laborales.

\begin{tabular}{|c|c|c|c|c|c|c|}
\hline Exigencias & Fatiga & $\begin{array}{l}\text { Psicosomáticos } \\
\text { digestivos }\end{array}$ & $\begin{array}{l}\text { Trastorno } \\
\text { mental }\end{array}$ & $\begin{array}{l}\text { Cefalea } \\
\text { migraña }\end{array}$ & $\begin{array}{l}\text { Trastorno } \\
\text { sueño }\end{array}$ & $\begin{array}{l}\text { Psicosomáticos } \\
\text { corazón }\end{array}$ \\
\hline Rotación de turnos & - & - & - & - & $\star \star \star$ & - \\
\hline Doble turno & - & * & - & * & * & - \\
\hline Mucha atención & $\star \star$ & $\star \star *$ & $\star \star$ & - & $\star \star$ & * \\
\hline Ritmo intenso & ** & ** & ** & - & * & - \\
\hline Demasiado trabajo & $\star \star \star$ & $\star \star \star$ & $\star \star \star$ & ** & $\star \star \star *$ & $\star \star$ \\
\hline Trabajo aburrido & $\star \star \star$ & $\star \star \star$ & $\star \star \star$ & $\star \star$ & $\star \star$ & - \\
\hline Supervisión estricta & $\star \star \star$ & $\star \star$ & * & * & $\star \star$ & ** \\
\hline Mucha responsabilidad & $\star \star$ & * & * & - & $\star \star \star$ & - \\
\hline Emergencias & $\star \star \star$ & $\star \star$ & $\star \star \star$ & $\star \star$ & * & - \\
\hline Trabajo peligroso & ** & * & ** & * & $\star \star \star$ & * \\
\hline Esfuerzo físico pesado & $\star \star$ & $\star \star \star$ & $\star \star \star$ & $\star \star$ & $\star \star$ & - \\
\hline Posiciones forzadas & $\star * \star$ & $\star \star \star$ & $\star \star \star$ & $\star \star *$ & $\star \star \star$ & - \\
\hline
\end{tabular}

$\mathrm{n}=830 ;{ }^{*} \mathrm{p}<0,05 ; * \star \mathrm{p}<0,01 ; * \star \star \mathrm{p}<0,001$.

Modelos logísticos, ajustados por edad y antigüedad.

Fuente: Encuesta Individual. 
Desde el enfoque de la epidemiología laboral entendemos por interacción la relación estadísticamente significativa entre dos o más exigencias. Dentro de estas interacciones, la más importante es la sinérgica que se entiende como la potenciación de la probabilidad de producir un efecto (trastorno o daño) cuando se conjuntan dos o más exigencias. Esta probabilidad será mayor que la suma de las probabilidades de cada una de las exigencias por separado.

Cuando no hay interacción entre las exigencias, puede producirse la aditividad, y por ésta entendemos que la probabilidad de producir un efecto es aproximadamente la suma

Tabla 4

Interacción ${ }^{1}$ sinérgica de exigencias asociadas con trastornos mentales.

\begin{tabular}{lc}
\hline Exigencias & Trastornos mentales \\
\hline Rotación de turno y doble turno & $\star \star$ \\
Rotación de turno y demasiado trabajo & $\star \star$ \\
Doble turno y mucha atención & $\star$ \\
Doble turno y ritmo intenso & $\star \star \star$ \\
Esfuerzo físico y trabajo peligroso & $\star \star$ \\
Demasiado trabajo y trabajo peligroso & $*$
\end{tabular}

1 Nivel de significancia. Modelos Logísticos. Ajustado por edad y antigüedad. ${ }^{\star} p<0,05 ;{ }^{* \star} p<0,01 ; * \star \star p<0,001$.

Fuente: Encuesta individual.

Figura 1

Interacción Sinérgica. Probabilidad* de trastornos mentales de los que rotan turno ante la presencia de demasiado trabajo.

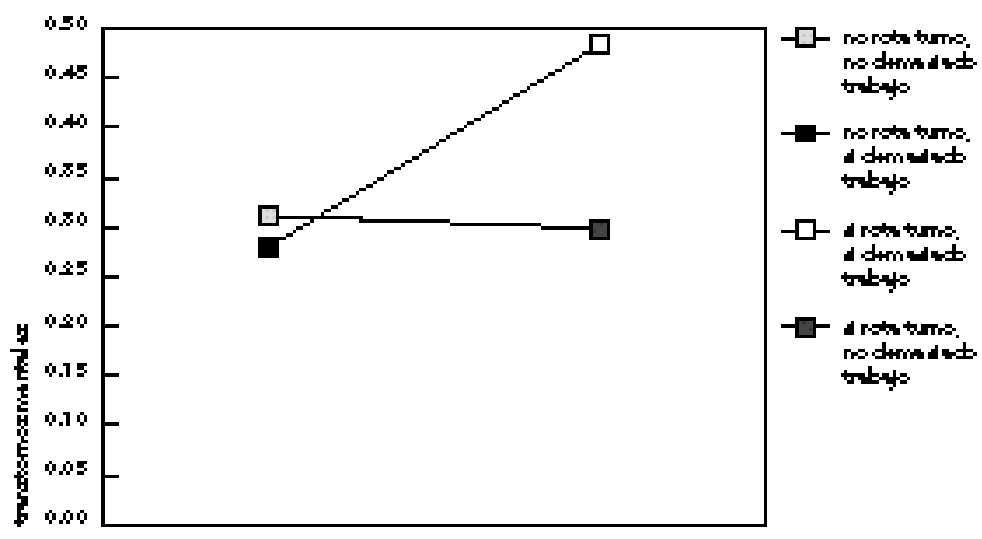

* Promedio de probabilidad ajustado por edad y antigüedad.

Fuente: encuesta individual. de la probabilidad de cada una de esas exigencias por separado.

Desde el punto de vista de la salud laboral, es importante tanto el sinergismo como la aditividad, pero obviamente también el conocimiento de aquellas exigencias que no producen ninguno de estos efectos, ya que esto significaría que no influyen en la aparición de esos daños o trastornos.

Un acercamiento inicial al efecto sinérgico de las exigencias en relación con los TMPF se muestra en el Tabla 4 y en la Figura 1; y el efecto aditivo se puede observar en el Tabla $5 \mathrm{y}$ en la Figura 2.

Resulta interesante constatar (Tabla 4) cómo los trabajadores expuestos a rotación de turnos presentan diferencias estadísticamente significativas para los trastornos mentales, en relación con los trabajadores no expuestos, cuando se encuentra interactuando con otras exigencias, situación que no se presenta cuando se analiza de manera desagregada, como ya se señaló en el Tabla 3.

En particular, los trastornos mentales se presentan asociados, en su gran mayoría, con aquellas exigencias que tienen que ver con el tiempo de trabajo (rotación de turnos y doble turno) en su interacción con exigencias que implican una sobrecarga cuantitativa, tales como el exceso de trabajo, la atención excesiva y el ritmo intenso. También llama la atención que la sobrecarga cuantitativa (esfuerzo físico intenso y demasiado trabajo) potencia el riesgo de producir trastornos mentales cuando las actividades laborales implican un trabajo peligroso.

Con la Figura 1, se ilustra el sinergismo a partir de la interacción de la rotación de turnos con demasiado trabajo, sobre la probabilidad de tener trastornos mentales. De esta manera, la probabilidad, ajustada por edad y antigüedad, de presentar este problema en quienes rotan turnos se incrementa en un $73 \%$, cuando se tiene demasiado trabajo. Este valor se calculó con base en la probabilidad de presentar trastornos mentales en aquéllos que rotan y tienen demasiado trabajo $(0,4834)$, en relación con quienes rotan y no tienen demasiado trabajo $(0,2789)$.

También se encontró que la interacción de la rotación de turnos con demasiado trabajo tiene un efecto sobre la aparición de trastornos digestivos y la fatiga, aunque no se menciona en la Tabla 4. Así, la probabilidad de tener este tipo de problemas de salud se incrementa (se potencia) cuando la rotación de turnos interactúa con el exceso de actividades a desarrollar. Este efecto sinérgico se presenta también en otras exigencias. Por ejemplo, la presencia 
Aditividad de exigencias asociadas con trastornos psicosomáticos y fatiga.

\begin{tabular}{|c|c|c|c|c|}
\hline \multirow[t]{2}{*}{ Exigencias } & \multicolumn{4}{|c|}{ Trastornos psicosomáticos } \\
\hline & Digestivos & Cefalea & Corazón & Fatiga \\
\hline Rotación de turno y posición incómoda & A & A & A & A \\
\hline Rotación de turno y emergencia & A & A & A & A \\
\hline Doble turno y mucha atención & A & A & A & A \\
\hline Doble turno y mucha responsabilidad & A & A & A & A \\
\hline Doble turno y ritmo intenso & A & A & A & A \\
\hline Posición incómoda y supervisión estricta & A & A & A & A \\
\hline Demasiado trabajo y trabajo peligroso & A & A & A & A \\
\hline
\end{tabular}

Fuente: Encuesta individual.

de doble turno, cuando hay rotación de turnos, incrementa la probabilidad de tener tanto trastornos mentales como cefalea y migraña; o la presencia de trabajo peligroso, cuando hay esfuerzo físico intenso, potencia el riesgo también de los trastornos mentales y las cefaleas.

Este análisis logístico plantearía, en un primer momento, la necesidad de reducir la cantidad de trabajo, o el ritmo intenso, o la prolongación de la jornada de trabajo (doble turno) que realizan aquéllos quienes rotan turnos, como parte de su actividad laboral, lo cual se perfilaría como una medida protectora contra la aparición de trastornos de esta naturaleza.

Muchas exigencias no interactúan entre sí, sin embargo, su efecto es de carácter aditivo, es decir, se suman o se añaden para producir un determinado trastorno. Éste es el caso de la gran mayoría de las exigencias estudiadas. La aditividad es sumamente frecuente en la generación de trastornos psicosomáticos y fatiga. Como se puede ver en la Tabla 5, todos lo pares de exigencias reportados sumaron su efecto para producir trastornos digestivos, cefalea y migraña, trastornos del corazón y fatiga.

Es interesante notar que los pares encontrados combinan, por lo general, como en el caso del sinergismo, exigencias relacionadas con el tiempo de trabajo (rotación de turnos y doble turno) con exigencias vinculadas con el tipo de tarea (posiciones incómodas y forzadas, emergencia, mucha atención, responsabilidad y ritmo intenso). Es claro que este conjunto de la Tabla 5 evidencia serios problemas en las formas de organización del trabajo y refuerza la relación estrecha que se establece entre la esfera fisiológica y la mental.

Esta situación se ilustra en la Figura 2, mediante la cual se muestra que la probabilidad de presentar trastornos digestivos, ante la pre-
Figura 2

Aditividad. Probabilidad* de trastornos digestivos en quienes doblan turno y tienen mucha atención.

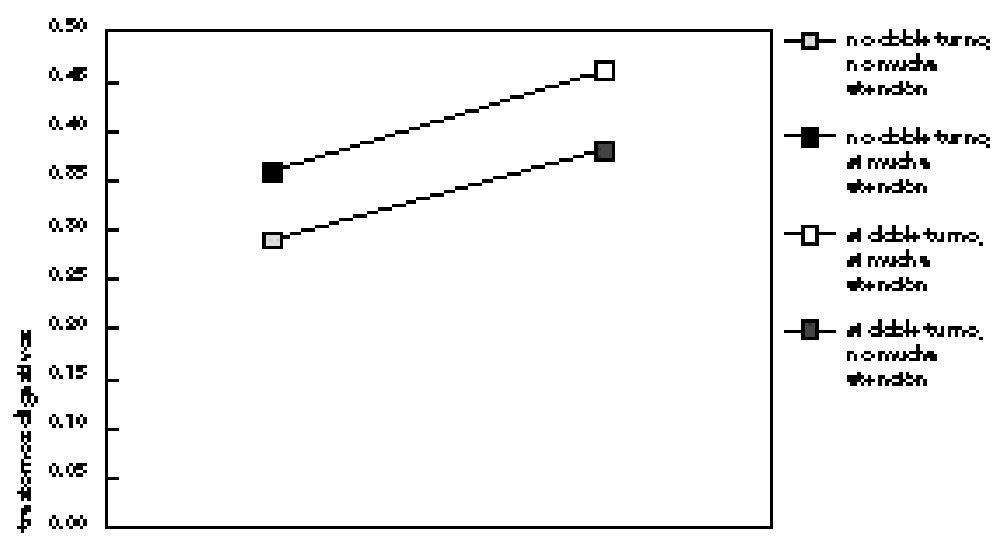

* Promedio de probabilidad ajustado por edad y antigüedad. Fuente: encuesta individual.

sencia de dos exigencias - mucha atención y doble turno - es mayor al efecto de cada una de ellas por separado. En el caso de esta gráfica, el efecto equivale aproximadamente a la suma de ambas.

\section{Conclusiones}

1) La pretensión del estudio fue demostrar que la relación entre el trabajo y la salud no se puede reducir a un análisis unicausalista o a enfermedades o trastornos aislados. Las diferentes categorías y variables aquí tratadas son las ma- 
nifestaciones específicas o las respuestas inmediatas del proceso global de trabajo. Las expresiones concretas del complejo trabajo siderúrgico son sus exigencias y trastornos. Es decir, lo que se ha querido captar son las diferentes manifestaciones de un problema global común.

2) En particular se estudiaron el tipo de actividad, el área de trabajo, la antigüedad en el puesto y algunas exigencias laborales a las que se someten estos obreros, tratando de demostrar la relación de ellas con los TMPF.

3) El énfasis de esta investigación se puso en la interacción de las exigencias laborales como generadoras de padecimientos psíquicos y psicosomáticos. El estudio de los efectos sinérgico y aditivo vislumbra la posibilidad de incursionar en un análisis más complejo de las causas que se encuentran en la base de este conjunto de cuadros patológicos, explorando con mayor detalle las características que adopta la organización del trabajo al interior de los centros laborales.

4) La explicación precisa de por qué interactúan estas exigencias entre sí y guardan una asociación estadística con los conjuntos patológicos estudiados, y porqué no interactúan otras exigencias, es un problema que puede estar en vías de empezar a resolverse. En todo ca- so, nuestro interés es dejar planteado un primer avance en este sentido y continuar su indagación a través de estudios futuros.

5) Es evidente la importancia cada vez mayor que representa el trabajo como determinante de muchos TMPF. La significación de estos trastornos en el conjunto del perfil patológico nos indica el énfasis que amerita esta cuestión. Las condiciones actuales en países como México, con un proceso de transformación económica de magnitudes enormes, que afecta especialmente a los trabajadores, tanto en limitar su control, organización y defensa de ciertas condiciones de trabajo, aparejado a las limitaciones salariales y de reproducción de los trabajadores, en general, pronostican situaciones laborales y de salud mental graves para estos trabajadores.

Se han realizado estudios que han encontrado la utilidad de emplear cuestionarios para seleccionar grupos de alto riesgo que requieren de la realización de acciones de intervención para mejorar los puestos de trabajo. Esta puede ser una de las utilidades de los estudios realizados aquí, ya que, al encontrar puestos de trabajo con multiexposición, se estarían identificando prioridades de intervención (Hildebrandt et al., 1996).

\section{Referencias}

ABRAMXON, J., 1964. The Cornell Medical Index as an Epidemiological Tool. Jerusalem: Hadasah University Hospital.

AKERSTEDT, T.; KNUTSSON, A.; ALFREDSSON, L. \& THEORELL, T., 1984. Shift work and cardiovascular disease. Scandinavian Journal of Work, Environment \& Health 10:409-414.

ALMIRALL, P. \& REYES, M. E., 1982. Relación entre índices subjetivos y objetivos de fatiga. Validación de una prueba. Revista Cubana de Higiene y Epidemiología, 20:239-248.

APA/OPS (American Psychiatric Association/Organización Panamericana de la Salud), 1988. DSM-IIIR. Manual Diagnóstico y Estadístico de los Trastornos Mentales. Barcelona: Masson Editores.

ARO, S. \& HASAN, J., 1987. Occupational class, psychological stress and morbidity. Annals of Clinical Research, 19:62-68. 
COUTO, H.; BASILE, Y.; DA LUZ, M. \& DOS SANTOS, F., 1981. Fadiga psíquica. Resultados da aplicação de um método alternativo para a identificação dos agentes etiológicos ligados ao ambiente de trabalho. Revista Brasileira de Saúde Ocupacional, 9:16-24.

CREED, F., 1993. Mental health problems at work. $B M J, 306: 1082-1083$.

GARDELL, B., 1971. Production technology, alienation and mental health: Summary and conclusions. In: Produktionsteknik och Arbetsglädje. Socialpsykologisk Studie av Industreillt Arbete (B. Gardell, ed.), pp. 375-391, Estocolmo: PA Radet.

GOLDBERG, P.; DAVID, S.; LANDRE, M. F.; GOLDBERG, M.; DASSA, S. \& FUHRER, R., 1996. Work conditions and mental health among prision staff in France. Scandinavian Journal of Work, Environment and Health, 22:45-54.

HENAO, S., 1982. Salud mental y trabajo. Revista Centroamericana de Ciencias de la Salud, 8:57-64.

HILDEBRANDT, V. H.; BONGERS, P. M.; DUL, J.; VAN DIJK, F. J. \& KEMPER, H. C. 1996. Identification of high-risk groups among maintenance workers in a steel company with respect to musculoskeletal symptoms and workload. Ergonomics, 39:232-242.

HOIBERG, A., 1982. Occupational stress and illness incidence. Journal of Occupational Medicine, 24: 445-451.

KARASEK, R. \& THEORELL, T., 1990. Healthy Work: Stress, Productivity, and the Reconstruction of Working Life. New York: Basic Books Publishers.

KAWAKAMI, N. \& HARATANI, T., 1999. Epidemiology of job stress and health in Japan: Review of current evidence and future direction. Industrial Health, 37:174-186.

KNUTSSON, A., 1986. Increased risk of ischaemic heart disease in shift workers. Lancet, 2: 89-92.
LAURELL, C.; NORIEGA, M.; LOPEZ, O. \& MARTINEZ, S., 1991. El trabajo como determinante de la enfermedad. Cuadernos Médicos-Sociales, 56:17-33.

RUEDA, I., 1998. La industria siderúrgica. In: La Privatización en México: Consecuencias Sociales y Laborales (E. de la Garza, org.), pp. 257-281, México, D.F.: Instituto de Estudios de la Revolución Democrática.

SANCHEZ, L. \& GONZALEZ, J., 1997. Cambio tecnológico, condiciones de trabajo perfiles de salud. Salud de los Trabajadores, 5:33-42.

SEGAWA, K.; NAKAZAWA, S.; KURITA, Y.; GOTO, H.; FUKUI, A. \& TAKANO, K., 1987. Peptic ulcer is prevalent among shift workers. Digestive Diseases and Sciences, 32:449-453.

SELIGMANN, E., 1986. Crise econômica, trabalho e saúde mental. In: Crise, Trabalho e Saúde Mental no Brasil (C. Costa, ed.), pp. 54-132, São Paulo: Editora Traco.

SHIGEMI, J.; MINO, Y.; TSUDA, T.; BABAZONO, A. \& AOYAMA, H., 1997. The relationship between job stress and mental health at work. Industrial Health, 35:29-35.

STOCKMEIER, U.; KUHN, T. \& MUTH, H., 1989. Stress at work and coronary heart diseases of foremen in industry. International Journal of Rehabilitation Research, 12:310-312.

WILCOSKY, T. \& WING, S., 1987. The healthy worker effect. Selection of workers and work forces. Scandinavian Journal of Work, Environment and Health, 13:70-72.

YANG, M. J.; HO, C. K.; SU, Y. C. \& YANG, M. S. 1997. Job strain, social support and mental health: A study on the male heavy manufacturing workers. Kaohsiung Journal of Medical Sciences, 13:332341.

YOSHITAKE, H., 1978. Three characteristic patterns of subjective fatigue symptoms. Ergonomics, 21: 231-233. 